\title{
Study of Functional Foods Consumption Patterns Among Decedents Dying Due to Various Causes of Death
}

\author{
Ram B. Singh*, ${ }^{*}$, Pradeep Visen ${ }^{2}$, Divyank Sharma ${ }^{1}$, Sarthak Sharma ${ }^{1}$, Ratin Mondol ${ }^{3}$, J.P. Sharma ${ }^{1}$, \\ Meena Sharma ${ }^{4}$, Miki Tokunaga ${ }^{5}$, Toru Takahashi ${ }^{5}$, Sanjay Mishra ${ }^{6}$, Akanshi Sharma ${ }^{7}$, Monika \\ $\mathrm{Jain}^{7}$, Fatima Marinho ${ }^{8}$ and Raina $\mathrm{Pal}^{9}$
}

${ }^{1}$ Halberg Hospital and Research Institute, Moradabad, India; ${ }^{2}$ Department of Nutritional Sciences, Faculty of Medicine, University of Toronto and Nutra-V Inc., Toronto, Canada; ${ }^{3}$ Hypertension Research Center, Rangpur, Bangladesh; ${ }^{4} \mathrm{~J} \mathrm{M}$ Hospital and Nursing Home, Seohara, Bijnore; ${ }^{5}$ Department of Nutritional and Health Sciences, Fukuoka Women's University, Fukuoka, Japan; ${ }^{6}$ Department of Biotechnology, IFTM University, Moradabad 244 102, (UP), India; ${ }^{7}$ Department of Food Science and Nutrition, Banasthali Vidyapith, Banasthali, India; ${ }^{8}$ Ministry of Health, Rio de Janeiro, Brazil; ${ }^{9}$ Hindu College, Moradabad, India

\begin{abstract}
Background: Western diet appears to be a risk factor for non-communicable diseases (NCDs), cardiovascular diseases (CVDs), diabetes and cancer whereas dietary supplements of functional foods rich Mediterranean diets are inversely associated, among individuals and populations with underlying lack of general and health education. We have only scanty information about functional foods which may be considered as nutraceuticals. The prevalence of optimal functional food eating behavior pattern in the population is unknown. This study examines the prevalence of optimal functional foods eating behavior as a protective factor among victims dying due to NCDs to find out the accuracy of the verbal autopsy questionnaire.
\end{abstract}

Subjects and Methods: Death records of 2,222 (1,385 men and 837 women) decedents, aged 25-64 years, out of 3,034 death records, were randomly selected and studied by verbal autopsy questionnaires. All the risk factors and protective factors were assessed by questionnaires which were completed with the help of the victim's spouse and a local treating doctor, by a trained scientist. The lack of knowledge on health education about the role of adverse effects of Western foods was assessed by the questionnaires. Functional food intake was considered in presence of fruit, vegetable and legume intake of minimum $250 \mathrm{~g} /$ day (moderate) and ideally $400 \mathrm{~g} / \mathrm{day}$ consistent with WHO guidelines.

Results: The prevalence of optimal prudent foods intake behavior; fruit, vegetable and legume (>250g/day) intake were observed among $51.4 \%(n=712)$ men and $50.4 \%(n=422)$ women. Western type food $(>255 \mathrm{~g} /$ day $)$ intake was observed among $63.2 \%(n=875)$ men and $59.9 \%(n=502)$ women. The prevalence of optimal functional food intake was significantly greater among men compared to women $(19.4 v s 14.6 \%, \mathrm{P}<0.05)$. The consumption of functional food not fruits; grapes and apples, guava, stargoose berry and lemon and onion, garlic ginger was significantly greater among men as compared to women. Other functional foods such as soy products, mustard or olive oil, curd or yogurt, nuts and fish, tea and cocoa, spices; turmeric, cumin, coriander seeds and peppers intake showed no significant difference between two sexes.

Multivariate logistic regression analysis revealed that after adjustment of age and body weight, total functional foods intakes and fruit, vegetable, legume and nuts intake were significantly inversely associated with deaths due to NCDs, whereas Western type foods (red meat and eggs, refined foods ) were positively associated with these causes of deaths, in both sexes. Total spices intake, mustard/olive oil intake and curd or yogurt intake, were inversely but weakly associated with causes of deaths due to NCDs, among both men and women. The prevalence of protective behavior pattern was observed among half of the victims, dying due to injury and accidents where such behaviors were uncommon among decedents dying due to NCDs.

Conclusions: It is possible that protective health behavior about functional food intake can be accurately assessed by a verbal autopsy questionnaire administered to a spouse of the decedents among Indians. The consumption of functional food appears to be lower among victims dying due to CVDs and cancer.

Keywords: Health behavior, health foods, diet and lifestyle, functional foods.

*Address correspondence to the author at the Department of Nutritional Sciences, Faculty of Medicine, University of Toronto, Canada;

Tel: 00915912417437; E-mail: rbs@tsimtsoum.net

\section{INTRODUCTION}

The risk factors of major non-communicable diseases (NCDs); (cardiovascular diseases (CVDs), type 2 diabetes, 
chronic respiratory diseases, cancer and degenerative diseases of brain) high blood pressure, and high cholesterol, tobacco and alcohol consumption, and obesity [1-3].

Proatherogenic Western diet appears to be an important pathway for the development of NCDs apart from these risk factors, among individuals and populations with underlying lack of general and health education [1-5]. The prevalence of prudent dietary intake and other health behaviors; physical activity, no tobacco and moderate alcohol intake in the population which are known to protect against NCDs, are not well known [6-11]. However, most agencies advise; prudent diet, regular moderate physical activity and cessation of tobacco and alcoholism for prevention of cardiovascular diseases (CVDs) and other chronic diseases [12-15]. Case control studies and intervention trials indicate that adherence to prudent dietary patterns in conjunction with other healthy lifestyle can protect against NCDs including CVDs [16-22]. Recently, functional foods such as components of Mediterranean style diets have been demonstrated to be protective against NCDs [7,16-22]. Hence, Mediterranean style foods and some of the Asian foods; fruits; grapes and apple (flavonoids, phytosterols, soluble fiber), guava and stargoose berry (vitamin C), crane berries (flavonoids), soya products (isoflavones), walnuts (omega-3 fatty acids and essential amino acids), flex seeds (omega-3 fatty acids), fish (omega-3 fatty acids), olive oil (antioxidant flavonoids and MUFA), and mustard oil (omega-3 fatty acids), bitter gourd (flavonoids), leafy vegetables and tea and cocoa (flavonoids) as well as spices like turmeric, cumin, coriander, peppers (flavonoids and minerals)fenugreeks may be considered as nutraceutical functional foods to highlight their role in the prevention of NCDs [16-32]. Functional foods are those foods which contain one or more protective nutrient that can address any biological mechanism in the body, in providing the benefit.

There is only little information about adoption of healthy diet and lifestyle behaviors among individuals with cardiovascular diseases and type 2 diabetes in communities across a range of countries worldwide. Such information about decedents dying due to NCDs, is unknown. In a recent study, association of high omega-6/omega-3 fatty acid ratio in the Mediterranean style diet with causes of death due to NCDs, has been observed among urban decedents in North India [33]. This study examines the association of Western foods consumption as well as functional food intake with risk of NCDs, among victims dying due to various causes of death.

\section{SUBJECTS AND METHODS}

Random death records of 2,222 (1,385 male and 837 female) decedents, aged 25-64 years, was studied by verbal autopsy questionnaires as reported in an earlier publication [33]. All the clinical manifestations, risk factors and protective factors were assessed by questionnaires which was completed with the help of the victim's spouse and a local treating doctor practicing in the concerned street. Causes of deaths were assessed by modified questionnaires based on WHO with greater emphasis on systemic manifestations by showing models or pictures of the brain, heart, lungs, liver and pancreas (Appendix I). The lack of knowledge on health behaviors; prudent diet and food consumption pattern and alcoholism was studied by the validated questionnaires. Subjects who admitted to drinking of alcohol at least 5-10 drinks per week on alternate days, were categorized as moderate alcohol-consuming.

Physical activity was assessed by questionnaire on occupational, household and spare time activity. Prudent dietary habits were considered in the presence of fruit, vegetable and legume intake of minimum $400 \mathrm{~g} /$ day (WHO), and moderate health foods intake when fruit, vegetable and legume intake was 250-400 g/day. No tobacco was considered when there was no past history of tobacco intake and no tobacco consumption during the last 30 days.

\section{Dietary Patterns and Functional Food Consumption}

Dietary intakes of the victim were obtained by trained interviewers by finding out the food intake of the spouse by using 3-day dietary diaries and filling of questionnaires, by asking probing questions about differences in food intake by the decedents (Appendix I). Food models, food measures and food portions were used by the trained scientist to find out the exact food intakes of the victims. Dietary intakes were also assessed with the use of a validated food frequency questionnaire that includes approximately 62 foods and beverages commonly consumed in India. Standard portion sizes were used for the estimation of consumed quantities, and functional nutrient content was assessed by using a food composition database modified to accommodate the particularities of the Indian diet [34]. For each participant, grams per day of intake of various food groups and nutrients, as well as total energy intake, were calculated. Salt intakes were assessed by finding out the amount of salt mixed in the food divided by the number of family members and then adding salt consumed by each member during eating. We focused on nine nutritional variables: fruits, vegetables, legumes, and nuts, milk and curd, cereals, meat/fish/ eggs.

\section{FUNCTIONAL FOOD INTAKE SCORE}

The traditional Indian diet is rich in legumes, seasonal vegetables, wheat and rice and seasonal fruits and nuts. Beef, meat, eggs and chicken are consumed by the Moslems, occasionally by others. Refined foods such as bread, biscuits, cookies and syrups have become common in the diets of social class 1-3. Milk, curd, cooking oils; soy bean, corn, mustard, sunflower, butter and Indian clarified butter are also common foods in the Indian diets. Since soy products; soy milk and soy pakori are also used commonly, these health foods have also been included in the Indian diet. Fish, almonds and walnuts are expensive items which are consumed by higher social classes. We considered grapes, apple, guava, stargoose berry, blue berries, soya products, walnuts, almonds, flex seeds, fish, curd/yogurt, olive oil, and mustard oil, bitter gourd, onion, garlic, ginger, leafy vegetables, tomato, tea, cocoa and red wine, as well as spices like turmeric, cumin, coriander, peppers as functional foods [16-34]. These foods are known to be rich in vitamins, flavonoids, minerals, omega-3 fatty acids as well as essential and nonessential amino acids [16-34]. 
The conformity of the functional food intake was studied with a 10 unit scale. The scale relies on 8 dietary components that capture the essence of functional foods in the traditional Indian diet. Fruits, vegetables and nuts, whole grains/legumes; (Bengal gram, kidney beans, peas, other beans) carrots, yogurt and curd, spices and mustard oil that are presumed to be beneficial for health. However, proatherogenic foods, such as red meat and eggs and butter, clarified butter, omega- 6 rich oils, trans fatty acids, sugar, salt, refined foods are presumed not to be beneficial. We assigned values of 0 or 1 to each of the above indicated components, using the sex specific medians in the studied population as cut-offs. We assigned a value of 0 to people whose consumption was below the median values of components with a presumably beneficial effect and a value of 1 to people with consumption equal to or above the median. In contrast, we assigned a value of 1 to people with below the median consumption of components without a beneficial effect and a value of 0 to those whose consumption of these components was above the corresponding median. Thus, the total diet score can take values from 0 (minimal conformity to the Functional food diet) to 9 (maximal conformity to the Functional food rich diet). The diagnosis of risk factors was based on available records, inquiry from the spouse and doctors involved as reported earlier [33].

\section{STATISTICAL ANALYSIS}

We used the chi-square test for the comparison of frequencies and two sample t test for longitudinal variables among men and women in the two groups. Only $\mathrm{P}$ values $<0.05$ and the two tailed t-test were considered significant to find out the level of significance and difference. Multivariate logistic regression analysis was conducted, after adjustment of age and body mass index, to demonstrate the association of functional foods with risk of death due to NCDs.

\section{RESULTS}

Demographic data are given in Table 1. Mean age, mean body weight and mean body mass index were significantly greater among men compared to women. The intake of functional food and alcohol intake was also more common among men as compared to women. Causes of deaths due to NCDs included CVDs ( $\mathrm{n}=695,31.3 \%)$, malignant neoplasm $(\mathrm{n}=131,5.8 \%)$, kidney diseases $(\mathrm{n}=250,11.3 \%)$, pulmonary diseases $(n=495,22.3 \%)$ and liver diseases $(n=107,4.8 \%)$. The total number of deaths due to NCDs was $58.3 \%$ $(n=1295)$ as reported earlier (33). The prevalence of protective behavior as regards to no tobacco was; $51.6 \%$ $(\mathrm{n}=715)$ among men and $83.9 \%(\mathrm{n}=702)$ among women victims. Fruit, vegetable and legume $(>250 \mathrm{~g} /$ day $)$ intake were observed among $51.4 \% \quad(\mathrm{n}=712)$ men and $50.4 \%$ $(n=422)$ women. The prevalence of functional food intake were significantly greater among men as compared to women (19.4 vs $14.6 \%, \mathrm{P}<0.05)$.

This modified verbal autopsy questionnaire allowed us to diagnose deaths due to NCDs among $56.4 \%(n=781)$ of men and $61.4 \%(n=514)$ women with a total of $58.3 \%$ (1295). This sum of deaths included $23.4 \%$ deaths due to heart diseases and $9.8 \%$ due to brain diseases, including stroke and inflammatory brain diseases and other chronic diseases. Renal diseases including acute renal failure and chronic renal failure were the cause of death among $11.2 \%$ of victims and other causes among 8.5\%. Accidental deaths according to modified questionnaires were much higher (14.0\%).Using the modified questionnaires, most of the victims could be classified relatively more accurately, into various causes of death according to body systems. Table $\mathbf{2}$ shows the functional food intakes among both sexes. The consumption of functional fruits; grapes and apples, guava, stargoose berry and lemon and onion, garlic ginger was significantly greater among men as compared to women. Other functional foods such as soy products, mustard or olive oil, curd or yogurt, nuts and fish, tea and cocoa, spices; turmeric, cumin, coriander seeds and peppers intake showed no significant difference between two sexes (Table 2). Total functional foods consumption was significantly higher among men than women.

Table 1. Clinical data and nutritional factors among decedents, based on records and answers given by spouse.

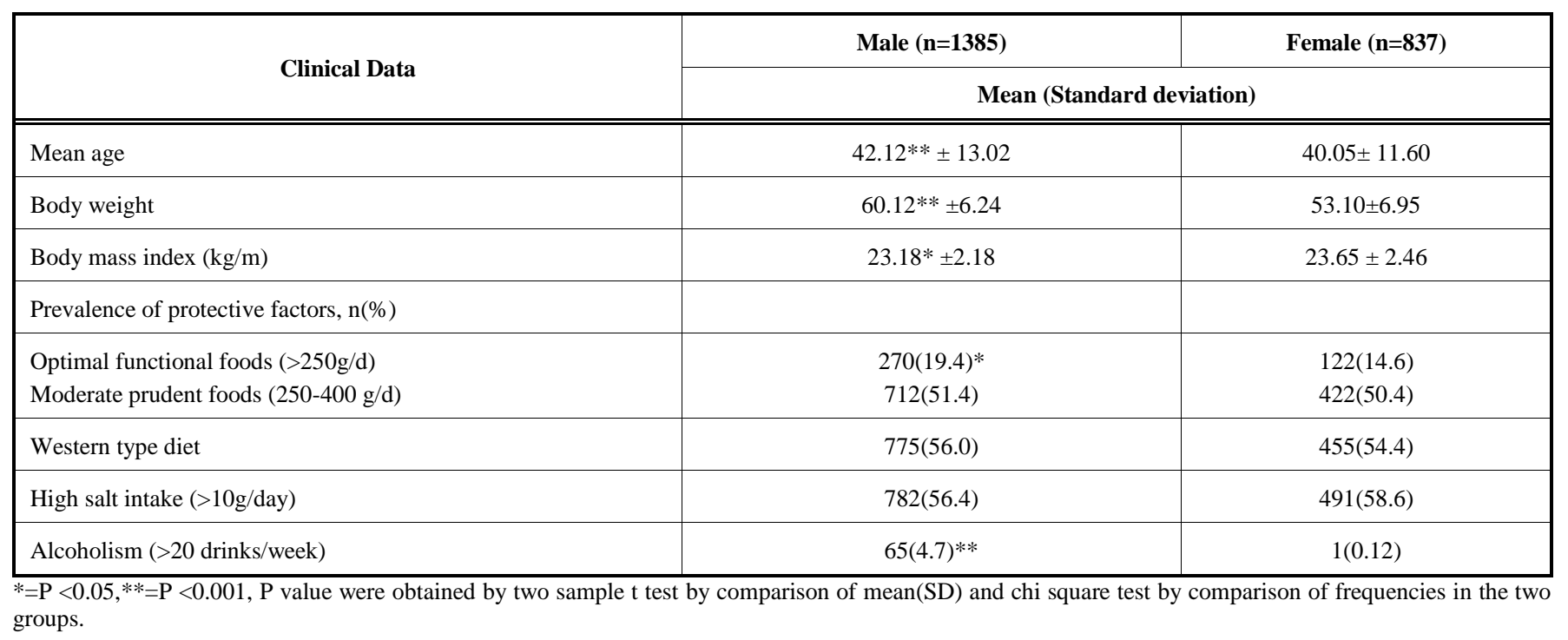


Table 3 shows the food consumption pattern among victims with various causes of death in relation to sex. Western type refined and proatherogenic foods (sugar, cola drinks, bread, biscuits, chocolates, cakes, refined cereals; corn flakes); clarified butter, butter, trans fat and omega-6 rich oils, meat and eggs and salt were also common food items consumed by both sexes. The consumption of prudent foods; whole grains, fruits, vegetables, legumes and nuts as well as milk and curd including functional foods was common in both men and women. Table $\mathbf{3}$ reveals that there is an overall decrease in the consumption of prudent foods including functional foods and an increase in the Western type proatherogenic foods among victims who died due to malignant, circulatory and infective causes of death compared to injury and accidents among both men and women. The functional food score also showed a significant decreasing trend among these decedents in both sexes. The trends for food intakes and functional food score were significant as revealed by the Kendall's i (tau) test.

Table 2. Functional food intake among victims dying due to various causes of death.

\begin{tabular}{|l|l|l|}
\hline \multicolumn{1}{|c|}{ Functional foods (g/day) } & \multicolumn{1}{|c|}{ Men $(\mathbf{n}=\mathbf{1 3 8 5})$} & \multicolumn{1}{|c|}{ Women $(\mathbf{n}=(\mathbf{8 3 7})$} \\
\hline \hline Grapes, apple, & $24.6 \pm 5.2^{*}$ & $20.2 \pm 4.6$ \\
\hline Guava, star goose berry, lemon, & $60.6 \pm 9.5^{*}$ & $53.6 \pm 9.1$ \\
\hline Bitter gourd, leafy vegetables, tomato, & $55.6 \pm 8.8^{*}$ & $50.2 \pm 7.8$ \\
\hline Onion, garlic, ginger & $22.8 \pm 4.6^{*}$ & $16.2 \pm 3.6$ \\
\hline Soya products, pulses & $10.6 \pm 2.2$ & $6.9 \pm 2.1$ \\
\hline Curd/yogurt, & $25.5 \pm 5.1$ & $21.3 \pm 4.7$ \\
\hline Walnuts, flex seeds, fish, pea nuts & $12.5 \pm 2.4$ & $10.6 \pm 2.0$ \\
\hline Tea, green /black and cocoa, & $5.3 \pm 1.2$ & $4.4 \pm 1.1$ \\
\hline Spices; turmeric, cumin, coriander, peppers, fenugreek & $5.6 \pm 1.2$ & $4.6 \pm 1.1$ \\
\hline Olive oil, and mustard oil, & $23.5 \pm 4.8$ & $20.2 \pm 4.1$ \\
\hline Red wine/moderate alcohol & $4.2 \pm 1.2$ & - \\
\hline Total functional foods & $250.8 \pm 35.5^{*}$ & $208.2 \pm 21.6$ \\
\hline$*=P<0.05$, by comparison of food intake in men and women by two sample t test. Valves are mean (Standard deviation)
\end{tabular}

$*=\mathrm{P}<0.05$, by comparison of food intake in men and women by two sample $\mathrm{t}$ test. Values are mean (Standard deviation)

Table 3. Food consumption pattern and functional food intakes, in relation to causes of death based on assessment by dietary diaries of the spouse and questionnaires filled by the nutritionist.

\begin{tabular}{|c|c|c|c|c|}
\hline Causes of death & Prudent foods & Proatherogenic foods & Functional foods & Score \\
\hline & \multicolumn{4}{|c|}{ Men(Mean \pm Standard deviation)g/day } \\
\hline $\begin{array}{l}\text { Miscellaneous+ injury } \\
\qquad(\mathrm{n}=270)\end{array}$ & $892 \pm 252$ & $202 \pm 22$ & $384 \pm 102$ & $7.95 \pm 2.2$ \\
\hline Malignant $(\mathrm{n}=77)$ & $715 \pm 241$ & $412 \pm 53$ & $217 \pm 76$ & $4.4 \pm 1.5$ \\
\hline Circulatory $(n=429)$ & $757 \pm 245$ & $437 \pm 47$ & $184 \pm 68$ & $4.82 \pm 1.6$ \\
\hline Infections $(n=609)$ & $806 \pm 237$ & $256 \pm 28$ & $168 \pm 58$ & $3.26 \pm 1.5$ \\
\hline \multirow[t]{2}{*}{ Total $(\mathrm{n}=1385)$ Kendall,s $\mathrm{l}$} & $0.045^{*}$ & $0.048^{* *}$ & $0.047 * *$ & $0.041 *$ \\
\hline & \multicolumn{4}{|c|}{ Women(Mean \pm Standard deviation)g/day } \\
\hline $\begin{array}{c}\text { Miscellaneous+ } \\
\text { injury }(n=211)\end{array}$ & $922 \pm 234$ & $86 \pm 23$ & $308 \pm 122$ & $7.42 \pm 2.3$ \\
\hline Malignant $(\mathrm{n}=54)$ & $657 \pm 197$ & $305 \pm 35$ & $212 \pm 71$ & $5.75 \pm 1.7$ \\
\hline Circulatory $(\mathrm{n}=266)$ & $655 \pm 205$ & $332 \pm 41$ & $181 \pm 76$ & $5.58 \pm 1.5$ \\
\hline Infections $(\mathrm{n}=306)$ & $736 \pm 237$ & $218 \pm 33$ & $154 \pm 82$ & $4.72 \pm 1.6$ \\
\hline Total $(\mathrm{n}=837)$ Kendall,s $\mathfrak{\imath}$ & $0.041^{*}$ & $0.067 * *$ & $0.065^{* *}$ & $0.043^{*}$ \\
\hline
\end{tabular}

Values are number mean (Standard deviation), $*=\mathrm{P}<0.01, * *=\mathrm{P}<0.001$ 
Multivariate logistic regression analysis revealed that after adjustment of age and body weight, total functional foods intakes and fruit, vegetable, legume and nuts intake was significantly inversely associated with deaths due to NCDs, whereas Western type foods (red meat and eggs, refined foods ) were positively associated with these causes of deaths, in both sexes. Total spices intake, mustard/olive oil intake and curd or yogurt intake, were inversely but weakly associated with causes of deaths due to NCDs, among both men and women.

\section{DISCUSSION}

Numerous studies have demonstrated that diets based on plant foods or Mediterranean style diets are protective against CVDs and other chronic diseases [14-20]. Nutrient contents, such as omega-3 fatty acids, flavonoids, fibre, protein and monounsaturated fatty acids that are rich in Mediterranean style diets, are also correlated to NCDs. The beneficial effects of Mediterranean style diets are determined by the presence of functional foods causing ingestion of bioactive nutrients, in these diets [7]. Functional foods are characterized by the presence of certain bioactive nutrients, the quality and content of which are important in providing the benefit. This study shows that the consumption of total functional foods as well as fruits; grapes, apple, guava, stargoose berry, vegetables and nuts were strongly and inversely associated with risk of death due to NCDs (Table 4). These foods are the rich sources of vitamins, antioxidant flavonoids, omega-3 fatty acids, phytosterols and minerals as well as essential and nonessential amino acids which are known to be protective against NCDs [17-39]. Several studies have shown that the ingestion of bioactive substances, such as omega-3 fatty acids (linolenic, eicosapentaenoic, and docosahexaenoic acids), soluble fibers (guar gum, psyllium, pectin, and oat products), and phytosterols (stanols and sterols) may be protective [7-39]. The Zutphen Elderly Study, reported that dietary antioxidant flavonoids were inversely associated with risk of CAD [40]. We also found that, Western type foods (red meat and eggs, refined foods) were positively associated with these causes of deaths, in both sexes [41]. Total spices intake, mustard/ olive oil intake and curd or yogurt intake, were inversely but weakly associated with causes of deaths due to NCDs, among both men and women (Table 4). Other studies have also reported that these foods have adverse effects on morbidity and mortality due to NCDs [4-9]. A more recent study showed the prevalence of avoidance or cessation of smoking, eating a healthy diet, and undertaking regular physical activities by individuals with a CAD or stroke event [8].

Prospective Urban Rural Epidemiology (PURE) survey of 153996 adults, aged 35 to 70 years, from 628 urban and rural populations in 3 high-income countries (HIC), 7 uppermiddle-income countries (UMIC), 3 lower-middle-income countries (LMIC), and 4 low-income countries (LIC) revealed interesting findings [8].

Of 7519 subjects with self-reported CAD or stroke around $18 \%$ continued to smoke; only $35.1 \%$ did high levels of work- or leisure-related physical activity, and $39.0 \%$ had healthy diets; $14.3 \%$ could not be motivated to follow any of the 3 healthy lifestyle behaviors and $4.3 \%$ could follow all 3 behaviours. Overall, $52.5 \%$ quit tobacco more in high income than lower income countries The interesting point is the levels of physical activity increased with rising income but the trend was not statistically significant because it depends on health education of the population. A lower prevalence of eating healthy diets was common in LIC compared to HIC $(25.8 \% v s 43.2 \%, 45.1 \%)$. It is clear that among a sample of patients with a CAD or stroke event from countries with varying income levels, the prevalence of healthy life style behaviors such as eating fruits and vegetables was low, with even lower levels in poorer countries.

Fruits and vegetables contain, over 600 carotenoid compounds (fat-soluble plant pigments), including lycopene, a-tocopherol, lutein, b-cryptoxanthin, zeaxanthin and betacarotene [17-50]. Of these carotenoids, beta-carotine is the most widely studied, which is the major vitamin A precursor and is carried in plasma and LDL particles. Carotenoids prevent lipid peroxidation by providing electrons to quench singlet oxygen. Lycopene is a potent antioxidant, the substance that gives tomatoes their red color, appears to be more strong than beta-carotene. Epidemiologic studies have correlated high tissue concentrations of lycopene with a

Table 4. Multivariate logistic regression analysis for association of functional foods with risk of death from non-communicable diseases, after adjustment of age and body weight among both sexes.

\begin{tabular}{|c|c|c|}
\hline Risk factor & $\begin{array}{c}\text { Men } \\
\text { (Odd,s ratio,(95\% confidence interval) }\end{array}$ & $\begin{array}{c}\text { Women } \\
\text { (Odds ratio,(95\% confidence interval) }\end{array}$ \\
\hline \hline Total functional foods & $1.12(1.07-1.19)^{* *}$ & $1.08(1.04-1.13)^{* *}$ \\
\hline Total fruits, vegetables, pulses, nuts & $1.06(1.01-1.11)^{* *}$ & $1.04(0.98-1.09)^{* *}$ \\
\hline Total Spices; turmeric, coriander, cumin, peppers, & $1.00(0.93-1.06)^{*}$ & $(0.96-1.08)$ \\
\hline Mustard oil/olive oil & $1.03(0.94-1.09)^{*}$ & $0.98(0.93-1.04)^{*}$ \\
\hline Western type foods & $0.88(0.98-0.81)^{*}$ & $-0.93(0.99-0.83)^{*}$ \\
\hline Curd/yogurt & $1.22(1.11-1.32)^{*}$ & $1.16(1.10-1.25)^{*}$ \\
\hline Tea and cocoa & $1.10(1.09-1.21)^{*}$ & $1.13(1.07-1.21)^{*}$ \\
\hline
\end{tabular}

$* \mathrm{P}$ value $<0.01, * *<0.001$. $\mathrm{OR}=$ Odds ratio. 
lower risk of myocardial infarction however, prospective, controlled trials have not yet been completed with lycopene. Tomato seeds also provide some flavanols in the tomato seed oil, which have antiplatelet effects. The EPIC-NL Study is a cohort of 40,011 men and women aged 20-70 years, with 10-15 years of follow-up [47]. Diet was assessed with a validated food frequency questionnaire and the MDS was based on the daily intakes of vegetables, fruits, legumes and nuts, grains, fish, fatty acids, meat, dairy, and alcohol. In 34,708 participants free of CVD at baseline, 4881 CVD events occurred, and 487 persons died of CVD. A two unit increment in MDS (range 0-9) was inversely associated with fatal CVD (HR: 0.78; 95\%CI: 0.69-0.88), total CVD (HR: 0.95 (0.91-0.98)), myocardial infarction (HR: 0.86 (0.79$0.93)$ ), stroke (HR: $0.88(0.78-1.00)$ ), and pulmonary embolism (HR: 0.74 (0.59-0.92)). The Mediterranean style diet was not related to incident angina pectoris, transient ischemic attack and peripheral arterial disease. It is possible that a better adherence to a Mediterranean style diet was more strongly associated with fatal CVD than with total CVD. Disease specific associations were the strongest for incident myocardial infarction, stroke and pulmonary embolism.

The diets of Homo sapiens and Homo economicus populations indicate that Mediterranean style diets contain high quantity of functional foods rich in omega-3 fatty acids and low in omega- 6 fatty acids apart from high content of fiber, flavonoids, polyphenolics, vitamins, antioxidants and minerals $[7,14,15,50,51]$. High omega-6/omega-3 ratio diets are associated with greater risk and low omega-6/omega-3 ratio diets with lower risk of CVDs and other chronic diseases [33,36]. In a randomized, controlled intervention trial, effects of guava intake on serum total and high density lipoprotein cholesterol, lipid peroxides levels and systemic blood pressures were studied among 120 patients with hypertension for 3 months [35].

This study revealed that inclusion of high fiber functional food guava, in the diet can decrease fat intake and modulate blood lipids. In this study, 61 group A and 59 group B patients with essential hypertension were administered guava fruit preferably before meals in a foods-to-eat approach rather than foods-to-restrict, in a randomized and singleblind fashion for 12 weeks [35]. Nutrient intakes including saturated and total fat were significantly decreased; carbohydrates, total and soluble fiber and vitamins and mineral intakes were significantly higher in group A than in group B at 12 weeks. There was a significant net decrease in serum total cholesterol (9.9\%), triglycerides $(7.7 \%)$ and blood pressures $(9.0 / 8.0 \mathrm{~mm} \mathrm{Hg})$ with a significant net increase in high-density lipoprotein cholesterol $(8.0 \%)$ after 12 weeks of guava fruit substitution in group A than in group B. By adding moderate amounts of guava fruit in the usual diet, changes in dietary fatty acids and carbohydrates may occur, providing significant amounts of soluble dietary fiber and antioxidant vitamin $\mathrm{C}$, potassium, flavanoids and minerals which are known to provide, lipid lowering, antihypertensive and antioxidant effects, without any adverse effects.

Effects of fat modified and fruits vegetable enriched diets on blood lipids and lipid peroxides in the Indian diet heart study among 621 high risk subjects revealed that diet can decrease blood lipids, blood glucose and oxidative stress and increase high density lipoprotein cholesterol [42]. Randomized, double-blind, placebo-controlled trial of fish oil and mustard oil in 360 patients with suspected acute myocardial infarction revealed that eating fish oil or mustard oil can cause significant decline in morbidity and mortality during a follow up of one year [16]. Randomized, single blind controlled trial with functional food rich diet among 406 patients of acute coronary patients revealed that beneficial effects were noted within six weeks, one year $[17,18]$. After two years, there was a significant decline in CVDs and total mortality which were associated with significant decline in the omega-6/omega-3 ratio of the diet [43]. The intervention group received significantly greater amount of functional foods, fruits; apple, grapes, guava, papaya, oranges, vegetables; bitter gourds, green leafy vegetables, cabbage, brinjal, carrots, ladyfingers, onion, garlic, ginger, nuts; almonds, walnuts in conjunction with 20-30 g/day of mustard oil compared to control group [43]. The Leon diet heart study including Mediterranean style diet and rapeseed oil margarine (given to 600 post heart attack patients) and the Indo-Mediterranean diet heart study including fruits, vegetables and mustard oil, given to 1000 high risk subjects also reported beneficial effects of functional food rich diets, (low in omega-6/omega-3 fatty acids) on morbidity and mortality among patients with high risk of CVDs $[36,44,45]$. The beneficial effects of this diet were attributed to decreased omega-6/omega-3 fatty acid ratio in the diets. Accruing evidence about benefits of adherence to Mediterranean diet on health have also been observed in an updated systematic review with meta-analysis showed that each two unit increment in the Mediterranean diet score was associated with a $10 \%$ lower incidence of CVD [46]. The meta-analysis combined studies with different fatal and nonfatal CVD endpoints. The PREDIMED trial enrolled a total of 7447 persons, aged 55 to 80 years including 57\% were women who were followed up for 4.8 years [48]. The two Mediterranean-diet groups had good adherence to the intervention, according to self-reported intake and biomarker analyses. A primary end-point event occurred in 288 participants. The multivariable-adjusted hazard ratios were 0.70 (95\% confidence interval [CI], 0.54 to 0.92 ) and 0.72 (95\% CI, 0.54 to 0.96 ) for the group assigned to a Mediterranean diet with extra-virgin olive oil (96 events) and the group assigned to a Mediterranean diet with nuts (83 events), respectively, versus the control group (109 events). No diet-related adverse effects were reported. Among persons at high cardiovascular risk, a Mediterranean diet supplemented with extra-virgin olive oil or nuts reduced the incidence of major cardiovascular events. The Sydney Diet Heart Study included 458 men aged 30-59 years with a recent coronary event [49]. The intervention group received replacement of dietary saturated fats (from animal fats, common margarines, and shortenings) with omega 6 linoleic acid (from safflower oil and safflower oil polyunsaturated margarine). Controls received no specific dietary instruction or study foods and all non-dietary aspects were designed to be equivalent in both groups. An intention to treat, survival analysis approach to compare mortality outcomes by group showed that the intervention group $(\mathrm{n}=221)$ had higher rates of death than controls $(\mathrm{n}=237)$ (all cause $17.6 \% \mathrm{v} 11.8 \%$, 
hazard ratio 1.62 (95\% confidence interval 1.00 to 2.64 ), $\mathrm{P}=0.05$; cardiovascular disease $17.2 \% \mathrm{v} 11.0 \%, 1.70$ (1.03 to 2.80), $\mathrm{P}=0.04$; CAD $16.3 \%$ v $10.1 \%, 1.74$ (1.04 to 2.92 ), $\mathrm{P}=0.04)$. Inclusion of these recovered data in an updated meta-analysis of linoleic acid intervention trials showed nonsignificant trends toward increased risks of death from CAD (hazard ratio 1.33 (0.99 to 1.79$) ; \mathrm{P}=0.06)$ and CVDs (1.27 (0.98 to 1.65$) ; \mathrm{P}=0.07)$. It is remarkable that substituting dietary linoleic acid in place of saturated fats increased the rates of death from all causes, as well as CAD, and CVD. An updated meta-analysis of linoleic acid intervention trials showed no evidence of cardiovascular benefit. These are the limitations of meta analysis and funded research. These findings indicate that this approach appears to be useful for the food industry to develop superfoods by further modifications of probiotics with omega-3 fatty acids, flavanols by adding cocoa, walnuts and black raisins.

A meta analysis of several prospective cohort studies, quantitatively assessed the relation between fruit and vegetable intake and incidence of $\mathrm{CAD}$ by carrying out a meta-analysis of cohort studies which reported relative risks (RRs) and corresponding 95\% confidence interval (CI) of CAD with respect to frequency of fruit and vegetable intake [51]. A total of 278459 subjects (9143 CAD events) with a median follow-up of 11 years were included in this study. The subjects who had less than 3 servings/day of fruit and vegetables, the pooled RR of CAD was 0.93 (95\% CI: $0.86-$ $1.00, \mathrm{P}=0.06)$ while those with more than 5 servings/day, the $\mathrm{RR}$ was $0.83(0.77-0.89, \mathrm{P}=0.0001)$. This meta-analysis demonstrated that increased consumption of fruit and vegetables, less than 3 to more than 5 servings/day is related to a $17 \%$ reduction, whereas increased intake to 3-5 servings/day is associated with a smaller and borderline significant reduction in CAD risk. These results provide evidence supporting that 5 or more servings per day of fruit and vegetables, can provide substantial protection from CVDs. A large cohort study [14] involving 72,113 female nurses who were free of CAD, stroke, diabetes, and cancer, factor analysis identified prudent and Western dietary patterns from data collected on serial food frequency questionnaires. During a follow-up of 18 years, 6011 deaths occurred (3319 [52\%] as a result of cancer; $1154(19 \%)$ resulting from CVDs; and 1718 (29\%) resulting from other causes. There was a $17 \%$ lower risk of total mortality among those who were most adherent to the prudent diet (highest versus lowest quintile of adherence), a $28 \%$ lower risk of CVD mortality, and 30\% lower mortality from non-CVD, non-cancer causes. Cancer was not associated with the inverse prudent dietary pattern. A comparison of the highest and lowest quintiles of adherence showed that consumption of the Western diet was associated with increased total mortality (21\%), CVD mortality (22\%), cancer mortality $(16 \%)$, and mortality from non-CVD, non-cancer causes (31\%). Hence, except for cancer, risk relationships for the prudent and Western dietary patterns appear to be the inverse of each other: thus, mortality was increased as adherence to the prudent diet decreased and adherence to the Western diet increased. In the INTERHEART study, participants from 52 countries were included $(n=A C S=5761$, controls=10,646) [6]. This study examined the relationship between dietary patterns and risk of acute coronary syndrome (ACS). They used Principal Component Analysis and identified 3 major dietary patterns; Oriental (high intake of tofu and soy and other sauces), Western (high in fried foods, salty snacks, eggs, and meat), and prudent (high in fruit and vegetables). Consistent with previous studies in single within-population cohort studies, the authors found an inverse association between the prudent pattern score and risk of ACS and a significant positive association between the Western pattern score and increased risk of ACS. No association of Oriental diet with risk of ACS was reported. A dietary risk score based on 7 food items on the food-frequency questionnaire (meat, salty snacks, fried foods, fruits, green leafy vegetables, cooked vegetables, and other raw vegetables) was constructed by the authors. The investigators found that even after adjustment for established coronary risk factors, a higher score, indicating a poor diet was strongly associated with ACS risk and the subjects in the highest quartile of the score had nearly 2-fold increased risk. Further sensitivity analyses revealed, a consistent association for the composite diet score between men and women and across different regions of the world (North America, Western Europe, Australia, Central Europe, Middle East, Africa, south Asia, Southeast Asia, China, and South America). On the basis of an arbitrary cut point of the score (top 3 quartiles versus the bottom quartile), the investigators estimated that $30 \%$ of myocardial infarction (MI) could be explained by unhealthy diets worldwide. The benefits of prudent dietary patterns in the INTERHAERT Study appear to be increased due to the intake of fruits, vegetables, and whole grains which are rich sources of functional nutrients.

In a systematic review of studies which included the results from 14 human studies (7 prospective, 3 crosssectional, 1 controlled, 3 case-control) and 13 animal studies, found that dietary omega- 6 to omega- 3 fatty acid ratio can influence brain composition [21], Alzheimer's disease pathology, and behavior (as according to animal studies), and revealed an association between the omega- 6 to omega- 3 ratio, cognitive decline, and incidence of dementia. This review supports growing evidence of a positive association between the dietary omega-6/omega-3 ratio and the risk of Alzheimer's disease. A recent study involving 3,457 children aged 8 to 15 years, out of which 354 had reduced birth weight [22]. Systolic blood pressure was found to be $1.1 \mathrm{~mm}$ $\mathrm{Hg}$ higher in those with reduced (less than 10th centile) compared with normal birth weight, and pulse pressure was significantly higher $(3.4 \mathrm{~mm} \mathrm{Hg})$ in children with reduced birth weight. Those with reduced birth weight who were in the highest tertile of EPA and DHA intake were found to have significantly lower systolic blood pressure $(-4.9 \mathrm{~mm}$ $\mathrm{Hg})$ and pulse pressure $(-7.7 \mathrm{~mm} \mathrm{Hg})$, than those with normal birth weight. These data are consistent with the hypothesis that long-chain omega- 3 fatty acids reduce blood pressure in those with impaired fetal growth. These findings indicate that omega-3 fatty acids appear to be important functional nutrient of functional foods.

\section{CONCLUSIONS}

It has been difficult to identify the appropriate bioactive substance for the development of new functional foods associated with CAD, because the results of many clinical studies are contradictory [7]. Castro et al. used the 
multivariate statistical approach known as principal component analysis, followed by a mixed model to process data obtained from a meta-analysis aimed at evaluating simultaneously the effect of ingestion of 1 of 3 types of bioactive substances ( $\mathrm{n}-3$ fatty acids, soluble fibers, and phytosterols on 1 or more of 4 biomarkers (plasma total cholesterol, triacylglycerol, LDL cholesterol, and HDL cholesterol). Independent variables; number of patients per study, dose, age, body mass index, and treatment length and dependent variables; percentage change in blood total cholesterol, LDL, HDL, and triacylglycerol from 159 studies and substudies were organized into a matrix. The original values were converted to linear correlation units, which resulted in a new matrix. The findings revealed that two principal components were enough to explain $63.73 \%$ and $84.27 \%$ of the variance in the independent and dependent variables, respectively. Phytosterols and soluble fibers had a hypocholesterolemic effect, whereas omega-3 fatty acids lowered triacylglycerol and increased total, LDL, and HDL cholesterol. The principal component analysis and mixed model showed that this behavior was independent of dose, number of patients per study, age, and body mass index but was associated with treatment length. One important limitation was that other protective nutrients; polyphenolics and flavonoids, essential and nonessential amino acids and other antioxidants were not considered.Thus, 8 cocoa (flavanols), omega-3 fatty acids and probiotics have been demonstrated to cause significant decline in morbidity and mortality in various trials [19-22]. Antioxidant activity and total phenolics, vitamins, minerals, in selected fruits, vegetables, pulses, grain products and spices have been reported in several studies [52-63]. Indian foods appear to be rich in phenolics and flavonoids which have been determined in 85 foods comprising of cereals, pulses, nuts, oilseeds, vegetables, fruits and beverages [62]. Total phenolics were measured biochemically and flavonoids were measured as a sum of quercetin, kaempferol, luteolin and pelargonidin. High flavonoid content (> $100 \mathrm{mg} / 100 \mathrm{gm}$ ) was present in tea, coffee, apple, guava, terminalia bark, fenugreek seeds, mustard seeds, cinnamon, red chili powder, cloves and turmeric. Medium levels (50-100 mg) were found in Indian gooseberry, omum, cumin, cardamom, betel leaf and brandy. Small but significant amounts were also present in fooditems of large consumption such as kidney beans, soyabeans, grapes, ginger, coriander powder, bajra and brinjal. In a further study, antioxidant activity is presented as the range of values for each of the food groups [63]. The foods studied had good amounts of polyphenolic compounds, and antioxidant activity although they belonged to different food groups [63]. A significant correlation was observed between antioxidant activity (DPPH and FRAP) and polyphenolic compounds in most of the Indian foods. Commonly used domestic methods of processing may not affect the activity of phenolic compounds of the foods studied in general. There is a need to educate the food industry and agriculture scientists to develop functional foods characterized with low glycemic index and optimal amount of functional nutrienrts; soluble fiber, vitamins, minerals, antioxidant flavonoids, essential and nonessential amino acids and a balanced ratio of omega-6/omega-3 fatty acids, similar to the notional Paleolithic diet $[14,15,17,50]$. In earlier studies, these foods and nutrients rich in the Paleolithic style and Mediterranean style diets have been found to be protective against NCDs [15-17,50].

In a more recent study, on resveratrol levels and all-cause mortality in older adults reported no benefit of this polyphenol during follow up of several years [64]. The researchers published nine-year follow-up data of 783 men and women aged 65 years and older living in two small towns in the Chianti wine-producing region of Tuscany. After nine years, $34.2 \%$ of the participants died. After adjustment for multiple variables, including age, sex, bodymass index, lipid levels, and comorbidities, among others, total urinary resveratrol concentrations were not associated with mortality. In a more recent study, of the 783 participants, 639 were free of cardiovascular disease at baseline [64]. Follow-up revealed that, $27.2 \%$ went on to develop CVDs; a greater level of resveratrol revealed that the incidence of CVD was $22.3 \%, 29.6 \%, 28.4 \%$, and $28.0 \%$ $(\mathrm{p}=0.44)$. Similarly, there was no association between resveratrol concentrations and cancer risk, nor there was any association with inflammatory markers such as C-reactive protein (CRP), tissue necrosis factor (TNF), interleukin-6, and interleukin-1ß.

A lower risk of metabolic syndrome has been observed in populations eating Western vegetarian diets rich in fiber, flavonoids and omega-3 fatty acids $[65,66]$. It seems that glycemic index (GI) and the balance of foods and nutrients in the diets can delay the vascular complications of diabetes [66]. Vegetarian diets such as Mediterranean style diet or Indo-Mediterranean diet which decrease the post-prandial glycemia appear to be prudent in the management of diabetes and CVDs. It is possible by adding high-quality protein and fat and low glycemic index foods [67-69]. Diets that are rich in fibres can also improve insulin resistance and postprandial plasma glucose, metabolism, CVD and type 2 diabetes [6569].

It seems that food products rich in bioactive agents may be used as nutraceuticals such as dietary fibre, omega-3 fatty acids, prebiotics, probiotics, antioxidant vitamins and flavonoids and other different types of herbal/ natural foods. Nutraceuticals may help in the inhibition of diabetes and CVD risk factors like obesity, hypertension, hypercholesteremia and atherosclerosis [65-69]. In brief, evidence is clear that nutritional supplements may be beneficial in the treatment or prevention of type 2 diabetes [66-69].

\section{FUTURE DIRECTIONS}

Changing lifestyles have led to an epidemic of NCD in low and middle income countries. These populations suffer two thirds of all NCD deaths due to cancer, cardiovascular and respiratory disease as well as diabetes [70,71]. Long term and expensive treatment, thus threatening patients' and nations' budgets which enhances the poverty [70,71]. It seems that strengthening and providing primary prevention, may be the most cost-effective instrument to fight against major risk factors; tobacco, alcohol abuse, physical inactivity and western style diets. Primary prevention, in the field of NCDs requires special multisectorial platforms for affordable health education and health promotion instruments like free gymnesiums in the hotels and motels, 
NCD drugs and diagnostics for neglected population in all countries. A change in agricultural policy, food and nutrition policy and education policy is desirable to produce health foods which are rich in above nutrients and can simulate Paleolithic foods. In this regard, short-term educational programmes have been found to be effective in improving lifestyle and health. A durable education strategy via schools and cost-saving policies supported by sustained large-scale media education programmes could be the starting point for a possible national programme on controlling NCDs in general [70,71].

A change in agricultural policy is desirable to produce foods which are rich in above nutrients and can simulate Paleolithic foods. Further efforts should be made to educate the people and industry on protective effects of wines and fruit juices and to consider adding nitric oxide activating agents like epicatechin and flavones as well as omega-3 fatty acids in alcohol to provide additional beneficial effects. Apart from food industry, agriculture should be made functional by developing functional foods, using plant breeding and genetic engineering to enhance protective nutrients in the foods.

In brief, our results indicate that extended verbal autopsy for the assessment of health behavior; functional food intake, physical activity, and causes of death of the victims, appears to be reliable and valid. Decreased intake of functional foods may be associated with CVDs and other chronic diseases. Further studies using these methods of extended verbal autopsy, in a large scale cohort study may be validated, in any country of the world.

\section{APPENDIX I}

\section{Dietary and Functional Food Autopsy Questionnaire}

The following questions are about the foods that spouse usually eat (Difference from victim's food intake to be noted)

Please give the number of days per week on which you usually eat various foods.

Ring the appropriate answer like this:

If you eat a food 7 days a week, then ring the 7 , thus:

(7) $654321 \mathrm{M} \mathrm{R}$

If you eat a food 3 days a week, then ring the 3 , thus:

7654 (3) $21 \mathrm{M} \mathrm{R}$

If you eat a food less than one day a week but more

than once a month, then ring $\mathrm{M}$, thus:

$7654321(\mathrm{M}) \mathrm{R}$

If you eat a food less than once a month or never,

then ring $\mathrm{R}$, thus:

$7654321 \mathrm{M}(\mathrm{R})$

CEREALS: Please Answer Every Question

Bread Number/day Thickness

12. White $7654321 \mathrm{MR}$

THICK: MEDIUM: THIN

CHAPATI: Number/Day

13. Paratha $7654321 \mathrm{MR}$ THICK: MEDIUM: THIN

14. Puree 7654321 MR THICK: MEDIUM: THIN

15. Chapati $7654321 \mathrm{MR}$ THICK: MEDIUM: THIN

16. Dalia $7654321 \mathrm{MR}$

(g/week)

Office use (g/day)

\section{ROOT TUBERS:}

17. Potatoes boiled, baked, mashed

$7654321 \mathrm{MR}$

18. Potatoes: Chips or fried from shop

$7654321 \mathrm{MR}$

19. Potatoes: Chips or fried curry from home

$7654321 \mathrm{MR}$

20. Sweet Potato

$7654321 \mathrm{MR}$

\section{LEGUMES:}

21. Pulse-Red gram, green gram, Bengal gram

$7654321 \mathrm{MR}$

22. Baked beans, lentils of Butter beans

$7654321 \mathrm{MR}$

23. Peas, Kidney beans

$7654321 \mathrm{MR}$

VEGETABLES/SPICES:

24. Radish

$7654321 \mathrm{MR}$ 
25. Cabbage

26. Cauliflower

27. Green leafy Vegetables (grilled/fried/salad)

28. Onion

29. Garlic

30. Chilli/peppers

31. Black pepper

32. Spices (total, g/day)

33. Brinjal

34. Carrots

35. Bitter gourd

36. Jack fruit

FRUITS: (g/week)

37. How many bananas eaten per week?

38. How many guavas eaten per week?

39. How many apples eaten per week?

40. How many oranges eaten per week?

41. How many grape fruit eaten per week?

42. How many musk melons eaten per week?

43. How many slices of melons eaten per week?

44. How many tomatoes eaten per week?

45. How many mangoes eaten per week?

46. How many other fruits eaten per week? (name)

47. How many slices of papaya eaten per week? (name)

MILK:

48. Do you take milk Y/N

49. If yes, what type of milk do you usually have (St-No-go)

50. How much milk (not skimmed or dried) do you drink a

day in tea or coffee in milky drinks and in cereals
(a) Less than $100 \mathrm{ml}$
(b) $100-200 \mathrm{ml}$
(c) $200-500 \mathrm{ml}$
(d) More than $500 \mathrm{ml}$

CURD: How much curd do you take

g.........../week,

FOOD HABIT- Vegetarian/non-veg./ eggitarian

EGGS and FISH

51. How many eggs do you eat per week?

52. Fish (Singhara/oily, fish fingers)

$7654321 \mathrm{MR}$

53. Which fish do you take (River fish/Sea fish /both)?

MEATS:

54. Lamb, curry, fried

$7654321 \mathrm{MR}$

55. Chicken or other poultry, curry fried
$7654321 \mathrm{MR}$

Number

$7654321 \mathrm{MR}$
$7654321 \mathrm{MR}$

$654321 \mathrm{M} \mathrm{R}$

$7654321 \mathrm{MR}$

$7654321 \mathrm{MR}$

$7654321 \mathrm{MR}$

$7654321 \mathrm{MR}$

$7654321 \mathrm{MR}$

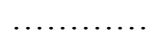

(g/week)

Office use (g/day)

\section{(g/week)}

Office use (g/day) 
56. Beef (including mined beef) curry, fried

57. Pork curry fried

FATS: Visible fat, (g/week)

58. How much of the following foods do the whole family and person concerned use on average per week? $(\mathrm{g})$

(Whole Family)/Week (Person Concerned/Week)

Clarified butter (ghee)

Butter

Lard and vegetable ghee/trans fat

Fat (specify)

Liquid vegetables oils;

Mustard oil

Ground nut/soyabean/corn/sun flower oil

Cream

Sugar

Salt

\section{CONFECTIONARY:}

59. Ice cream, sweet yoghurt or chocolate

60. Digestive biscuits or plain biscuits

61. Milk sweet (Rasgula, Barfi)

62. Non milk sweets (Jalebi, Laddu)

69 . How many persons normally eat in your household?

Number of adults (including yourself)

Number of children 1-4 years old

70. Are you on a special diet?

No, If yes, then

(a) Slimming diet suggested by your doctor

(b) Diabetic diet

(c) Vegetarian diet

(d) Fruit and vegetable enriched diet

71. What type of milk do you usually have?

(a) Whole Cow's, Buffalo, Goat's, Mixes (packet)

(b) Skimmed

(c) Other (Specify please)

72. How many times per week you eat and drink outside

73. Do you eat heavy breakfast $(>1000$ Cal.)...... Dinner $(>1500 \mathrm{Cal}) \ldots($ Yes/No $)$

\section{How much functional foods you eat in the breakfast, snack, lunch and dinner? (g/day)}

a. Grapes, apple,

b. Guava, star goose berry, lemon,

c. Soya products, pulses/legumes

d. Walnuts, flex seeds, fish,

e. Curd/yogurt,

f. Olive oil, and mustard oil,

g. Bitter gourd, leafy vegetables, tomato, h. Onion, garlic, ginger

i. Tea, green /black and cocoa,

j. Red wine,

k. Spices; turmeric, cumin, coriander, peppers, fenugreek.

\section{Functional foods intake Score, 0-9}

Modified from reference 33 . 


\section{CONFLICT OF INTEREST}

Conflict of interest has not been declared by the authors.

\section{ACKNOWLEDGEMENTS}

International College of Nutrition and International College of Cardiology for logistic support to conduct this study.

\section{REFERENCES}

[1] 2013 AHA/ACC guidelines on lifestyle management to reduce cardiovascular risk: A report of the American College of cardiology and American Heart Association task force on practice guidelines. Circulation published online November 12 , 2013,http://circ.ahajournals. International College of Nutrition and International College of Cardiology, Jan 12, 2014.

[2] The "Heart Disease and Stroke Statistics - 2014 Update" Circulation 2014; 129: e28-e292.

[3] Moodie R, Stuckler D, Monteiro C, et al. Profits and pandemics: prevention of harmful effects of tobacco, alcohol, and ultraprocessed food and drink industries. Lancet 2013; 381: 670-9.

[4] Bantle JP. Dietary fructose and metabolic syndrome and diabetes. J Nutr 2009; 139: 63-8.

[5] Stuckler D, Nestle M. Big food, food systems, and global health. PloS Med 2012; 9(6). e1001242.

[6] Iqbal R, Anand S, Ounpuu S, et al. Dietary patterns and the risk of acute myocardial infarction in 52 countries: results of the INTERHEART study. Circulation 2008; 118: 1929-37.

[7] Castro IA, Barroso LP, Sinnecker P. Functional foods for coronary heart disease risk reduction: a meta-analysis using a multivariate approach. Am J Clin Nutr 2005; 82: 32-40.

[8] Teo K1, Lear S, Islam S et al. Prevalence of a healthy lifestyle among individuals with cardiovascular disease in high-, middleand low-income countries: The Prospective Urban Rural Epidemiology (PURE) Study. JAMA 2013; 309(15): 1613-21.

[9] Taylor AW, Price K, Fullerton S. A survey to assist in targeting the adults who undertake risky behaviours, know their health behaviours are not optimal and who acknowledge being worried about their health. BMC Public Health 2013; 13: 120.

[10] Tokunaga M, Takahashi T, Singh RB, et al. Diet, Nutrients and Non-communicable Diseases. Open Nutra J 2012; 5: 146-59.

[11] Singh RB, Fedacko J, Vargova V, et al. Singh's verbal autopsy questionnaire for assessment of causes of death, social autopsy, tobacco autopsy, and dietary autopsy based on medical records and interview. Acta Cardiol 2011: 66; 471-8.

[12] Cecchini M, Sassi F, Lauer J, Lee YY, Guajardo-Barron V, Chisholm D. Tackling unhealthy diets, physical inactivity, and obesity: health effects and cost-effectiveness. Lancet 2010;376: 1775-84.

[13] Singh RB, Fedacko J, Pella D, et al. Prevalence and risk factors of pre-hypertension and hypertension in five Indian cities. Acta Cardiol 2011; 66: 29-37.

[14] Heidemann C, Schulze MB, Franco OH, Van Dam RM, Mantzoros $\mathrm{CS}$, Hu FB. Dietary patterns and risk of mortality from cardiovascular disease, cancer, and all causes in a prospective cohort of women. Circulation 2008; 118: 230-237.

[15] Gardener H, Wright CB, Gu Y, et al. Mediterranean style diet and risk of ischemic stroke, myocardial infarction and vascular death : the Northern Manhattan Study. Am J Clin Nutr 2011; 94: 1458-64.

[16] Singh RB, Niaz MA, Sharma JP, Kumar R, Rastogi V, Moshiri M. Randomized, double-blind, placebo-controlled trial of fish oil and mustard oil in patients with suspected acute myocardial infarction: the Indian experiment of infarct survival-4. Cardiovasc Drugs Ther 1997; 11: 485-49.

[17] Singh RB, Rastogi SS, Verma R, Bolaki L, Singh R, Ghosh S. An Indian experiment with nutritional modulation in acute myocardial infarction. Am J Cardiol 1992; 69: 879-85.

[18] Singh RB, Rastogi SS, Verma R, et al. Randomized, controlled trial of cardioprotective diet in patients with acute myocardial infarction : results of one year follow up. BMJ 1992; 304: 1015-9.
Hristova K, Nakaoka T, Otsuka K, et al. Perspectives on chocolate consumption and risk of cardiovascular diseases and cognitive function. Open Nutra J 2012 ; 5: 207-12.

[20] Barraud D, Bollaert PE, Gibot S. Impact of the administration of probiotics on mortality in critically ill adult patients: A metaanalysis of randomized controlled trials. Chest 2013;143: 646-55.

[21] Loef M, Walach H. The omega-6/omega-3 ratio and dementia or cognitive decline: a systematic review on human studies and biological evidence. J Nutr Gerontol Geriatr 2013; 32: 1-23.

[22] Skilton MR, Raitakari OT, Celermajer DS. High intake of dietary long-chain $\omega-3$ fatty acids is associated with lower blood pressure in children born with low birth weight NHANES 2003-2008. Hypertension 2013; 61(5): 972-6.

[23] Racchi M, Daglia M, Lanni C, Papetti A, Govoni S, Gazzani G. Antiradical activity of water soluble components in common diet vegetables. J Agric Food Chem 2002; 50(5):1272-17.

[24] Turkmen N, Sari F, Velioglu YS. The effect of cooking methods on total phenolics and antioxidant activity of selected green vegetables. Food Chem 2005; 93(4): 713-18.

[25] Chipurura B, Muchuweti ZM, Manditseraa F. Effects of thermal treatment on the phenolic content and antioxidant activity of some vegetables. Asian J Clin Nutr 2010; 2(3): 93-100.

[26] Bajpai M, Mishra A, Prakash D. Antioxidant and free radical scavenging activities of some leafy vegetables. Int J Food Sci Nutr 2005; 56(7): 473-81

[27] Kuti JO, Konuru HB. Antioxidant capacity and phenolic content in leaf extracts of tree spinach (Cnidoscolus spp.). J Agric Food Chem 2004; 52(1): 117-21.

[28] Bunea A, Andjelkovic M, Socaciu C, et al. Total and individual carotenoids and phenolic acids content in fresh, refrigerated and processed spinach (Spinacia oleracea L.) Food Chem 2008; 108(2): 649-56.

[29] Sreeramulu D, Raghunath M. Antioxidant activity and phenolic content of roots, tubers and vegetables commonly consumed in India. Food Res Int 2010; 43(4): 1017-1020.

[30] Singh PP, Sharma P. Antioxidant basket: do not mix apples and oranges. Indian J Clin Biochem 2009; 24(3): 211-4.

[31] Dong M, He X, Rui HL. Phytochemicals of black bean seed coats: isolation, structure elucidation, and their antiproliferative and antioxidative activities. J Agric Food Chem 2007; 55(15): 6044-51.

[32] Vijaya KRC, Sreeramulu D, Raghunath M. Antioxidant activity of fresh and dry fruits commonly consumed in India. Food Res Int 2010; 43(1): 285-8.

[33] Fedacko J, Vargova V, Singh RB, et al. Association of high w-6/w3 fatty acid ratio diet with causes of death due to noncommunicable diseases among urban decedents in North India. Open Nutra J 2012; 5: 113-23

[34] Narsingrao BS, Deasthale YG, Pant KC. Nutrient Composition of Indian Foods. Indian Council of Medical Research, New Delhi 1989.

[35] Singh RB, Rastogi SS, Singh R, Ghosh S, Niaz MA. Effects of guava intake on serum total and high density lipoprotein cholesterol levels and systemic blood pressures. Am J Cardiol 1992; 70: 1287-91.

[36] Singh RB, Dubnov G, Niaz MA, et al. Effect of an IndoMediterranean diet on progression of coronary disease in high risk patients: a randomized single blind trial. Lancet 2002, 360: 145561.

[37] Singh RB, Fadecko J, Pellad D, De Meester F, Moshiri M, Aroussy WE. Superfood dietary approaches for acute myocardial infarction. World Heart J 2010; 2: 13-23.

[38] Ross S. Functional foods: the food and drug administration perspective. Am J Clin Nutr 2000; 71: 1735-8.

[39] Hicks KB, Moreau RA. Phytosterols and phytostanols: functional food cholesterol busters. Food Technol 2001; 55: 63-7.

[40] Hertog MGL, Feskens EJM, Kromhout D, et al. Dietary antioxidant flavonoids and risk of coronary heart disease: the Zutphen Elderly Study. Lancet 1993; 342: 1007-11.

[41] Rohrmann S, Overvad K, Bueno-de-Mesquita H B, et al. Meat consumption and mortality. Results from the European prospective investigation into cancer and nutrition. BMC Med 2013; 11: 63.

[42] Singh RB, Rastogi SS, Niaz MA, Ghosh S, Singh R. Effects of fat modified and fruits vegetable enriched diets on blood lipids in the Indian diet heart study. Am J Cardiol 1992; 69: 869-74.

[43] Singh RB, Fedacko J, Vargova V, Pella D, Niaz MA, Ghosh S Effect of low W-6/W-3 fatty acid ratio Paleolithic style diet in 
patients with acute coronary syndromes: A randomized, single blind, controlled trial, World Heart J 2012; 4: 71-84.

[44] De Lorgeril M, Renaud S, Mamelle N, et al. Mediterranean alphalinolenic acid-rich diet in secondary prevention of coronary heart disease. Lancet 1994; 343(8911): 1454-9.

[45] De Lorgeril M, Salen P, Martin JL, Monjaud I, Delaye J, Mamelle $\mathrm{N}$. Mediterranean diet, traditional risk factors and the rate of cardiovascular complications after myocardial infarction .Final report of the Lyon Diet Heart Study. Circulation 1999; 99: 779-85.

[46] Sofi F, Abbate R, Gensini GF, Casini A. Accruing evidence about benefits of adherence to Mediterranean diet on health: an updated systematic review with meta-analysis. Am J Clin Nutr 2010; 92(5): $1189-96$.

[47] Hoevenaar-Blom MP, Nooyens ACJ, Kromhout D, et al. Mediterranean Style Diet and 12-Year Incidence of Cardiovascular Diseases: The EPIC-NL Cohort Study. PLoS One 2012; 7(9): e45458.

[48] Ramón E, Emilio R, Jordi SS, et al. Primary prevention of cardiovascular disease with a Mediterranean diet. N Engl J Med 2013; 368: 1279-90.

[49] Ramsden CE, Zamora D, Leelarthaepin B, et al. Use of dietary linoleic acid for secondary prevention of coronary heart disease and death: evaluation of recovered data from the Sydney Diet Heart Study and updated meta-analysis. BMJ 2013; 346: e8707.

[50] Singh RB, Takahashi T, Nakaoka T, et al. Nutrition in transition from Homo sapiens to Homo economicus. Open Nutra J 2013; 6: 617.

[51] He FJ, Nowson CA, Lucas M, MacGregor GA. Increased consumption of fruit and vegetables is related to a reduced risk of coronary heart disease: meta-analysis of cohort studies. J Hum Hypertension 2007; 21: 717-28.

[52] Rochfort S, Panozzo J. Phytochemicals for health, the role of pulses. J Agric Food Chem 2007; 55(20): 7981-94.

[53] Sreeramulu D, Reddy CVK, Raghunath M. Antioxidant activity of commonly consumed cereals, millets, pulses and legumes in India. Indian J Biochem Biophys 2009; 46(1): 112-5.

[54] Velioglu YS, Mazza G, Gao L, Oomah BD. Antioxidant activity and total phenolics in selected fruits, vegetables, and grain products. J Agric Food Chem 1998; 46(10): 4113-7.

[55] Arcan I, Yemenicioğlu A. Antioxidant activity and phenolic content of fresh and dry nuts with or without the seed coat. J Food Comp Anal 2009; 22(3): 184-8.

[56] Gupta S, Prakash J. Studies on Indian green leafy vegetables for their antioxidant activity. Plant Food hum Nutr 2009; 64(1): 39-45.
[57] Nair S, Nagar R, Gupta R. Antioxidant phenolics and flavonoids in common Indian foods. JAPI 1998; 46(8): 708-10.

[58] Murthy CKN, Singh RP, Jayaprakasha GK. Antioxidant activities of grape (Vitis vinifera) pomace extracts. J Agric Food Chem 2002; 50(21): 5909-14.

[59] Singh RP, Murthy CKN, Jayaprakasha GK. Studies on the antioxidant activity of pomegranate (Punica granatum) peel and seed extracts using in vitro models. J Agric Food Chem 2002; 50(1): 81-6.

[60] Aoshima H, Tsunoue H, Koda H, Kiso Y. Aging of whiskey increases 1,1-diphenyl-2-picrylhydrazyl radical scavenging activity. J Agric Food Chem 2004; 52(16): 5240-4.

[61] Matthäus B. Antioxidant activity of extracts obtained from residues of different oilseeds. J Agric Food Chem 2002; 50(12): 3444-52.

[62] Nagar R, Gupta R. Antioxidant phenolics and flavonoids in common Indian foods. JAPI 1998; 46:708-10.

[63] Sreeramulu D, Reddy CV, Chauhan A, Balakrishna N, Raghunath M. Natural antioxidant activity of commonly consumed plant foods in India: effect of domestic processing. Oxid Med Cell Longev 2013; 2013: 12.

[64] Semba RD, Ferrucci L, Bartali B, et al. Resveratrol levels and allcause mortality in older community-dwelling adults. Intern Med 2014; (7): 1077-84.

[65] Grooms KM, Omarborne MJ, Fan do Q, Djousse L, Clarke CR. Dietary fibre intake and cardiometabolic risks among US adults, NHANES 1999-2010. Am J Med 2013; 126: 1059-67.

[66] Rizzo NS, Sabate J, Jaceldo-Siegl K, Fraser GE. Vegetarian dietary patterns are associated with a lower risk of metabolic syndrome. Diabetes Care 2011; 34(5):1225-7.

[67] Das L, Bhaumik E, Raychudhary U, Chakraborty R. Role of Nutraceuticals in human health. J Food Sci Technol 2012; 49(2): 173-83.

[68] Massaro M, Scoditti E, Carluccio MA, De Caterina R. Nutraceuticals and prevention of atherosclerosis: focus on omega-3 polyunsaturated fatty acids \& Mediterranean diet polyphenols. Cardiovasc Ther 2010; 28(4): e13-9.

[69] Lee T, Dogoua JJ. Nutritional supplements and their effect on glucose control. Adv Exp Med Biol 2012; 771: 381-95.

[70] Bonita R, Beaglehole R. Women and NCDs: overcoming the neglect. Glob Health Action 2014; 7: 23742.

[71] Kontis V, Mathers CD, Rehm J, et al. Contribution of six risk factors to achieving the $25 \times 25$ non-communicable disease mortality reduction target: a modelling study. Lancet 2014; 384: 427-37. 